\title{
Impact of Position and Orientation of RFID Tags on Real Time Asset Tracking in a Supply Chain
}

\author{
Sidney D'Mello ${ }^{1}$, Eric Mathews ${ }^{2}$, Lee McCauley ${ }^{3}$ and James Markham ${ }^{4}$ \\ ${ }^{1}$ Department of Computer Science, University of Memphis, sdmello@memphis.edu \\ ${ }^{2}$ Mercury Technology Labs, Memphis, TN, USA eric@mercurytechlabs.com \\ ${ }^{3}$ Department of Computer Science, University of Memphis, mccauley@memphis.edu \\ ${ }^{4}$ FedEx Institute of Technology, University of Memphis, jmarkham@memphis.edu
}

Received 26 July 2007; received in revised form 6 January 2008; accepted 18 January 2008

\begin{abstract}
We studied the characteristics of four commercially available RFID tags such as their orientation on an asset and their position in a three dimensional real world environment to obtain comprehensive data to substantiate a baseline for the use of RFID technology in a diverse supply chain management setting. Using RFID tags manufactured by four different vendors and a GHz Transverse Electromagnetic (GTEM) cell, in which an approximately constant electromagnetic (EM) field was maintained, we characterized the tags based on horizontal and vertical orientation on a simulated asset. With these baseline characteristics determined, we moved two of the four tags through a real world environment in three dimensions using an industrial robotic system to determine the effect of asset position in relation to the reader on tag readability. Combining the data collected over these two studies, we provide a rich analysis of the feasibility of asset tracking in a real world supply chain, where there would likely be multiple tag types. We offer fine grained analyses of the tag types and make recommendations for diverse supply chain asset tracking.
\end{abstract}

Key words: Supply Chain Management, Tag Orientation on Asset, Asset Position, Multiple Tag Types 


\section{Introduction}

Total supply chain visibility is the key application of Radio Frequency Identification (RFID) technology. This means that as an asset or product travels through the supply chain, its attributes and sourcing information are associated with it. Attributes can include handling information, checkpoints or waypoint documentation, and chain of custody data as the asset moves between locations, processes, and exchanges hands. Ideally, the data would actually precede the arrival of the asset and also flow backward. Information preceding arrival gives each handler a forward view of the supply chain. Data that flows backward through the supply chain shows the lifecycle of the asset all the way back to the original manufacturer or material suppler. When this ideal data flow occurs, we call this total supply chain visibility. It is this visibility that benefits the entire supply chain from a command and control perspective. Real time, autonomously gathered, RFID data enables this control through total asset visibility up and down the supply chain.

However, although RFID technology has been proclaimed by many to be the next generation barcode, the critical challenges involved with widespread implementation of this technology have tempered adoption. Financial, security, and technical concerns have hindered wide use and acceptance of RFID technology in the supply chain management arena, as well as business, military, and medical areas [8], [18], [19], [24], [29], [32]. For many industries, the financial overhead associated with the technology has dramatically reduced adoption [6]. According to current cost estimates, tags purchased in bulk ( $>1$ million units) can cost about 13 cents each [1], whereas the current working alternative, barcodes, are virtually free. With improvements in manufacturing and further economies of scale in the future, RFID will likely become a cost effective solution for supply chain asset tracking. On the security and privacy front, the use of RFID has also been met with apprehension from consumers [13], [15]. Fortunately, security has been the focus of much of the ongoing research within the RFID community and innovative solutions to alleviate some of the security challenges have been proposed [9], [15], [12], [27].

Beyond these obstacles that can be overcome with more time and active research, the fundamental aspect of RFID technology that can truly hinder adoption includes the substantive challenge of reading tags in a real world environment. Simply put, state of the art RFID systems do not offer adequate asset tracking capabilities, at least when compared to barcodes. Additional complications arise in scenarios when a reader would need to optimally read multiple commercially available tag types operating in the same environment to ensure total asset visibility. It is these fundamental technical obstacles that motivate this study because it is our position that these factors will ultimately determine the success or failure of RFID technology with respect to its practical applicability in real world supply chains.

\section{Literature Review}

The introduction of RFID into the supply chain arena yields many complexities [3], [5], [21], [28]. Of particular importance is the need for industry standards, which is arguably a major factor that has severely limited broad acceptance of this technology. For small and mid-size companies, it can be hard to settle on a particular tag type or interrogator (reader, scanner), when one cannot be certain that the tags will be readable by other entities up or down the supply chain. On the other hand, small companies have the advantage of being able to experiment with RFID technology and fully implement a solution without the tremendous outlay of capital required of larger companies [22]. As will be shown below, the need for standardization goes beyond technology compatibility; it also includes standardizing on reader and tag placement. Another setback has come from technology vendors that promise better performance than their products achieve in real-world settings [4], [28].

The ultimate acceptance of RFID technology in supply chain management lies in reliably reading from and writing to RFID tags. While the performance of commercially available RFID systems such as tags, readers, and antennas have been meticulously calibrated in controlled settings of a sterile research lab, there are a host of additional issues that arise when tags and readers are deployed in real world settings. These include reader collisions when signals mutually collide [10], signal attenuation issues when tags are far away from a reader, and other environmental factors such as the presence of metallic components, cell phones, computer equipment, water sources, and people. Furthermore, even more germane to supply chains are other issues such as the location of the reader in space, the relative position of the RFID tags from the reader in three dimensional real world environments, and the orientation of the tags on assets [7], [11], [14], [23], [33], [35]. It is primarily these performance challenges that need to be overcome to enable adoption, not the financial or security concerns. As a result, the field is ripe for additional research documenting the nature of these problems coupled with innovative solutions to alleviate the major performance issues associated with writing to and querying RFID tags [12], [17], [20], [26], [31], [34].

At this time, the literature on highly detailed performance evaluations of RFID technology is sparse and scattered among a few studies with varied methods. The lack of available data on the real world performance of RFID systems has led several organizations within the supply chain management community to adopt a slap-and-ship approach to deploying tags on assets, as opposed to more informed tag deployment strategies that are sensitive to tag performance in the real world. 
Therefore, we set out to provide an unbiased evaluation of real world factors that affect readability of RFID tags. Furthermore, we wished to examine multiple tag types, document their characteristics in an effort to envision a diverse supply chain where multiple tags types would converge and need to be visible to all reader types. It is under these "real world" scenarios that we provide analyses to inform future work and adoption.

\section{Research Objectives and Approach}

Our research agenda involved an assessment of the effects of signal frequency and signal strength on tag readability. By investigating the optimal set of frequencies for a variety of commercially available RFID tags, querying algorithms could be improved such that readers could be tuned to pick up many tag types in the same environment - a benefit to diverse multi-source, multi-destination supply chains. Signal strength was investigated by attenuating the signal (i.e. no attenuation, strongest signal) in order to learn valuable information on how distance between the tag and the reader will impact its reading error rate. Again this characteristic varies by tag vendor.

Beyond these primary influences, a critical issue for RFID in supply chain settings relates to the tag's placement on an asset and the asset's relationship to the reader. By manipulating orientation of the tags by rotating the tags along horizontal and vertical axes, we can better understand how to reduce reading error rates in the supply chain. While ideally, tags should be orientation-invariant, i.e. readable at all orientations, the reality is that knowing the preferable orientations of tags on assets can enhance performance. Understanding the performance of RFID tags under varying orientations is critical for real world applications because it is impossible to pre-specify the orientation of a tag with $100 \%$ certainty particularly within a multiple-entity supply chain.

Consequently, we conducted a study (Study 1) where we characterized four RFID tags manufactured by three different vendors with respect to their horizontal and vertical orientation on a simulated asset. The distance between the asset and the reader was also investigated by attenuating the signal sent by the RFID scanner to query the tags. In order to establish suitable performance baselines for a variety of tags, we must look at their behavior in environments free of external electromagnetic (EM) interference. For example, in order to systematically understand the relationship between read error rates and tag orientation along a vertical axis, it is imperative that all other variables be held constant. Therefore, all tags in Study 1 were characterized in a $\mathrm{GHz}$ Transverse Electromagnetic (GTEM) cell, in which an approximately constant EM field was maintained.

Beyond tag orientation on an asset, other environmental factors such as the presence of metallic components, cell phones, computer equipment, water sources, and people, impact performance of RFID in the real world. While the GTEM cell provided us with an ideal scenario required for Study 1, such an environment can never truly exist in the supply chain. Therefore, we conducted a second study on tag readability where we moved two of the four tags through a real world environment in three dimensions using an industrial robotic system to determine the effect of asset position in relation to the reader. By assessing tag readability in such an environment and varying such factors as the tags' position in a three dimensional space and the frequency of the signal utilized by the reader to interrogate the tags, we can determine how tags deviate from the ideal and how differing tag types could potentially be interrogated in the same space with low error rates. With this knowledge in hand, we can make recommendations on supply chain adoption.

In this paper, we describe these studies that manipulated all these factors to provide a realistic view of RFID in real world supply chains. From the characteristics of RFID tags, to their orientation on an asset, to their position in three dimensional real world environments, we provide comprehensive information to inform future RFID technology development and adoption. We conclude by making recommendations for diverse supply chain asset tracking.

\section{Study 1: RFID Tag Characterization in a GTEM Cell}

\subsection{Motivation}

The motivation behind this study was to obtain a characterization of a variety of RFID tags within the closed environment of an RF chamber. In particular we were interested in exploring the effect that the orientation of the tags has on the associated read error rates. The orientation of the tags was manipulated by individually rotating the tags along a horizontal and a vertical axis. Understanding the performance of RFID tags under varying orientations is critical for real world applications because it is impossible to pre-specify the orientation of a tag. In essence, tags should be orientation-invariant (i.e. readable at all orientations).

We also investigated the impact of the signal strength on tag readability. This was accomplished by varying the degree of attenuation of the signal (i.e. no attenuation, strongest signal). The motivation behind varying the attenuation of the signal was to simulate distance between the tag and the reader. 


\subsection{Experimental Setup}

\subsubsection{Apparatus}

We carried out the study in an RF-chamber ( $\mathrm{GHz}$ Transverse Electromagnetic or GTEM cell), which is a radiated immunity and emission test facility that reduces the impact of the ambient electromagnetic environment. The GTEM cell was equipped with an octagonal turntable upon which the equipment under test (RFID tags in this case) can be securely mounted (see Figure 1A). The table top could be automatically rotated by use of an external controller with respect to an azimuthal (horizontal rotation, see Figure 1B) and an inclined orthogonal $\left(45^{\circ}\right)$ as shown in Figure 1. Since the turntable did not possess the capability of rotating vertically we designed and manufactured a vertically rotatable mount that could be manually positioned to a predefined angle (see Figure $1 \mathrm{C}$ ). The mount was designed such that a Styrofoam block that hosted a tag could be affixed to it and the entire block could be manually rotated in 5 degree increments. The materials used to construct the mount were Plexiglas and the various components were fused together with nylon bolts, hex nuts, and washers in order to minimize sources of metallic interference. In all experimental conditions, the tags were mounted on Styrofoam blocks and placed at the center of the turntable.

A. Tag in GTEM Cell

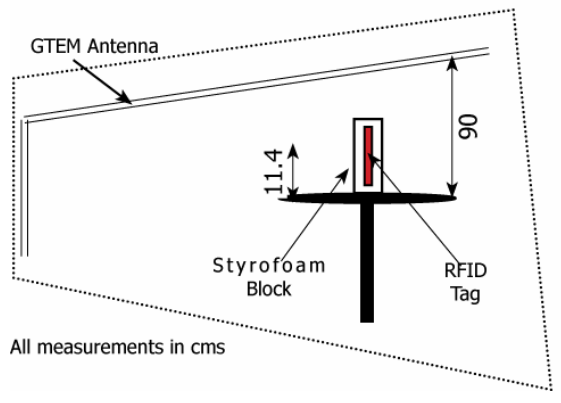

B. Horizontal Rotation

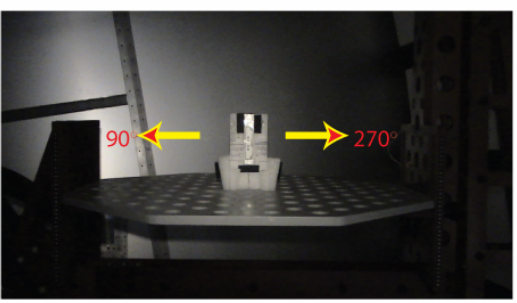

C. Vertical Rotation

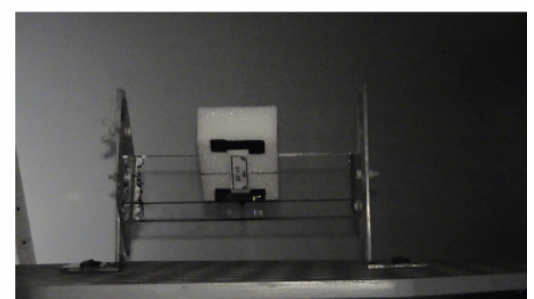

Figure 1: Experimental Setup

An Alien $^{\text {TM }} 9780$ RFID scanner [2] was used as the signal source in the RF chamber. The scanner sweeps frequencies over a $902-928 \mathrm{MHz}$ range in the ISM band. However, since one of the goals of this project was to investigate the frequency characteristics of the various tags (not elaborated in this article), the scanner was modified so that instead of automatically sweeping through all frequencies, a target frequency for transmission and reception of the signal could be set. Therefore, in the subsequent analyses we test tag reliability by incrementally setting the frequency over the $902-927 \mathrm{MHz}$ range.

The scanner is equipped with an internal attenuator that can cause a $0 \mathrm{dBm}$ to $16 \mathrm{dBm}$ attenuation of its output signal. The scanner is also equipped with an external ALR-9610-BC antenna. However, in the subsequent analyses the antenna of the GTEM cell was used for wireless communication with the tags bypassing the ALR-9610-BC.

Four EPC UFH Class 1 tags were used for the experiments. Three of the tags were commercially available RFID tags manufactured by Avery Dennison $\AA$ (AD-610 and AD-410) and Alien Technology $(A L-9338-02)$. The fourth was a prototype tag developed by an unspecified vendor. Throughout this report these tags will be designated as Tag-A for the unspecified tag, Tag-B for the AD-610, Tag-C for the AL-9338-02, and Tag-D for the AD-410 tag.

A Dell ${ }^{\mathrm{TM}}$ PC was used as a host computer that was connected to the turntable controller and a spectrum analyzer with a National Instruments ${ }^{\mathrm{TM}}$ USB-GPIB adapter. It was also connected to the RFID scanner through an Ethernet connection. The host computer also connected to the modified scanner in order to control the frequency via a parallel port. The scanner was controlled by the Alien Gateway ${ }^{T M}$ software version 3.3 installed on the host computer. An Application Programming Interface (API) written in the Java ${ }^{\mathrm{TM}}$ programming language was used for automated communication with the scanner.

\subsubsection{Procedure}

In the GTEM we measured the error rates obtained in an attempt to read the tags in the EM chamber. In particular this study involved an assessment of the effect of manipulating four independent variables on tag read errors (the dependent variable). The four independent variables used in this study consisted of the horizontal and vertical orientations of the tag in the chamber, the strength of the source signal, and the frequency of the source signal. Horizontal and vertical orientations were manipulated across a $0^{\circ}$ to $330^{\circ}$ range (full circle) with $30^{\circ}$ increments. For the vertical rotations a preliminary analysis resulted in expanding the scope to include the following angles: 40,50 , $130,140,220,230,310$, and 320. The output signal from the scanner was attenuated from $0-9 \mathrm{dBm}$ with $0.5 \mathrm{dBm}$ increments. Preliminary analyses confirmed that none of the tags could be read when the signal was attenuated beyond $9 \mathrm{dBm}$. The frequency of the signal was modulated across the $903-927 \mathrm{MHz}$ range with $1 \mathrm{MHz}$ increments. 
The RFID tag under test was mounted on a Styrofoam block and placed on the center of the turntable. The rotations across the horizontal and vertical axes were independent of each other. Specifically, when the tag was being horizontally rotated, its vertical angle was set to $0^{\circ}$ and vice versa. For each orientation of the tag on the turntable, for each attenuation setting, and for each frequency, 20 read attempts were performed.

\subsection{Results}

The four tag types were characterized on the basis of performance when rotated horizontally (horizontal directivity) and vertically (vertical directivity). We also evaluated performance when the signal was attenuated (sensitivity). The analyses proceeded by first computing an error rate for each read attempt for each tag. The error rate was computed as the ratio of the number of failures to the number of read attempts $(N=20)$. Therefore, the error rate ranges from 0 (perfect read) to 1 (complete failure).

Recall that for each orientation of the tag in the chamber, the modified scanner was used to incrementally set the frequency of the output signal across the $903-927 \mathrm{MHz}$ range. Additionally, for each frequency setting the output signal was attenuated across a $0-9 \mathrm{dBm}$ range with increments of $0.5 \mathrm{dBm}$. For each frequency, tag performance was calculated on the basis of the amount of attenuation required to obtain an error rate of approximately 0.5 . Higher attenuation rates imply good readability whereas lower attenuation rates are indicative of poor readability. This procedure formed the basis for computing the directivity and frequency characteristics of the RFID tags.

\subsubsection{Horizontal Directivity}

Figure 2A presents assessments of the horizontal directivity. It should also be noted that the data collection procedure was replicated for 3 individual tags from each of the four tag types (Tag-A, Tag-B, Tag-C, and Tag-D) yielding 12 runs in all. The results presented for each tag type have been averaged over the 3 individual tags. In Figure 2A we also average across the $903-927 \mathrm{MHz}$ frequency set.

\section{A. Horizontal Rotation}

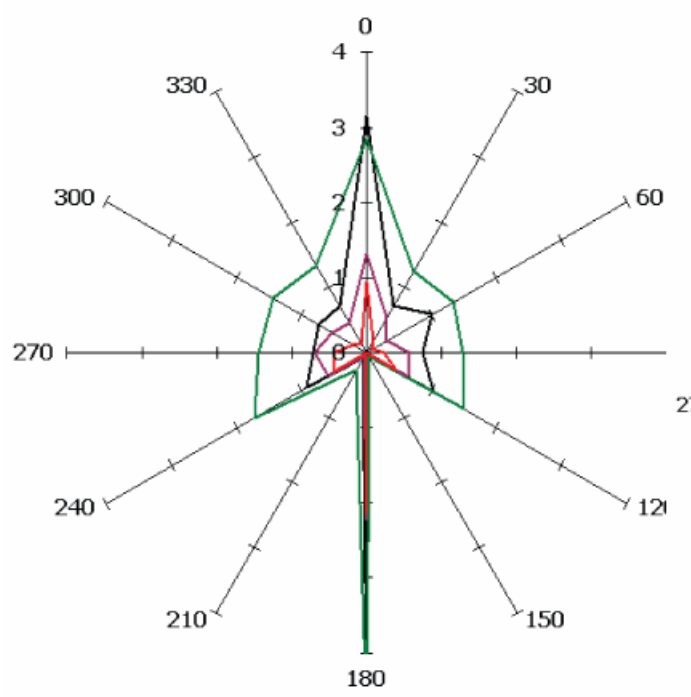

$\operatorname{Tag} \mathrm{A}$

\section{B. Vertical Rotation}

Figure 2: Average horizontal and vertical directivity. Circular axes represent the orientation. $Y$ axis is the attenuation required (in $\mathrm{dBm}$ ) to obtain an error rate of 0.5 averaged across the $902-903 \mathrm{MHz}$ frequency range

A number of general conclusions can be drawn on the basis of the mean horizontal directivity (Figure 2A). In particular performance of the four tags can be ordered as Tag-B, Tag-A, Tag-D, and Tag-C. Additionally, although performance of each tag type varies, a consistent trend in horizontal directivity emerges. Specifically, the best performance was obtained for the $0^{\circ}$ and $180^{\circ}$ orientations in which it takes about a $5 \mathrm{dBm}$ attenuation to cause a 0.5 reduction in performance (for Tags $\mathrm{A}$ and $\mathrm{B}$ ). The horizontal directivity was lower but remained consistent across the $30^{\circ}-120^{\circ}$ and $240^{\circ}-330^{\circ}$ ranges. The four tag types appear to have a $120^{\circ}$ blind spot between the $120^{\circ}-240^{\circ}$ range where they did not respond at all.

\subsubsection{Vertical Directivity}

Figure 2B depicts the mean vertical directivity. The results indicate a change in tag performance when compared to the horizontal directivity in that Tag-C outperforms Tag-D. Additionally, although Tag-A typically outperforms Tag-B, we observe Tag-B exhibiting a superior performance when rotated to the angles of $50^{\circ}, 130^{\circ}, 230^{\circ}$, and $320^{\circ}$. Similar 
to the results for the horizontal directivity a consistent pattern in terms of vertical directivity across all four tag types is observed. In particular, performance is the best for the $0^{\circ}$ and $180^{\circ}$ orientations and moderate for the $30^{\circ}, 150^{\circ}, 210^{\circ}$, and $330^{\circ}$ orientations. Performance then sharply drops across the $30^{\circ}-50^{\circ}, 130^{\circ}-150^{\circ}, 210^{\circ}-230^{\circ}$, and $310^{\circ}-330^{\circ}$ ranges. Finally, when the tags are extensively rotated $\left(50^{\circ}-130^{\circ}\right.$ and $\left.230^{\circ}-310^{\circ}\right)$ performance drops to 0 as noted by the fact that a non-attenuated signal produces no valid reads (an error rate of 1 ).

\subsubsection{Sensitivity}

The sensitivity for a tag provides an assessment of the error rate as a function of signal attenuation. Here we assume that the attenuation approximates the distance between the tag and the signal source (the transmitting antenna). Similar to the frequency characteristics described above the sensitivity analysis involved assessing the error rates associated with 5 individual tags of each type without rotating the turntable $\left(0^{\circ}\right.$ horizontal and $0^{\circ}$ vertical). On the basis of the sensitivity of each tag depicted in Figure 3 (which has been averaged across the 5 individual tags) we conclude that the sensitivity of Tag-A and Tag-B are on par and quantitatively higher than that of Tags $C$ and $D$ which are highly similar with each other. The results indicate that all tags break down and yield error rates of 1 when the level of attenuation exceeds 8 or $9 \mathrm{dBm}$. There also appears to be a linear relationship between the attenuation of the signal and the error rate of the tags. When averaged across the four tag types, a linear regression analysis yielded an excellent fit to the data with $\mathrm{R}^{2}=.94$.

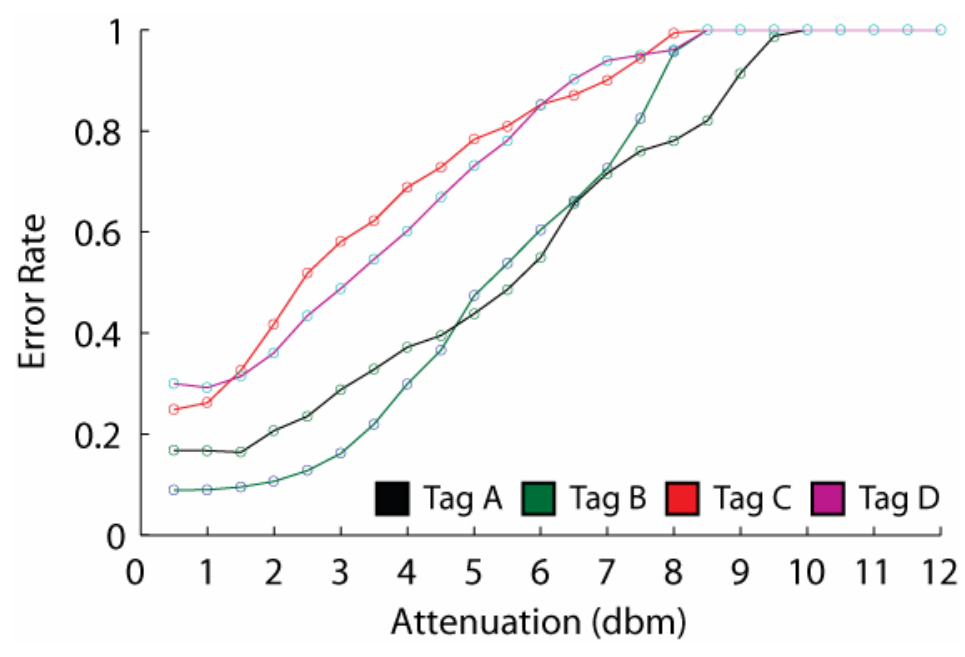

Figure 3: Sensitivity - Error rates as a function of signal attenuation

In general the results indicated that there are informed differences in the performance of the tags. In a multi-tag supply chain setting, we can see that error rates as a function of distance is an important factor when placing readers or working with suppliers and customers to determine the best tags for assets that flow through the supply chain through multiple organizations. As also demonstrated by the data, tag orientation on the asset is just as important a factor to specify in the supply chain for interrogation by a RFID reader. If a supplier sets a tag optimally for interrogation by his own readers and the acquiring vendor is not aware of this and changes reader orientation by 90 degrees the acquiring vendor effectively alters the signal and can increase the error rate by up to $100 \%$.

\section{Study 2: RFID Tag Characterization in a Simulated Supply Chain}

\subsection{Motivation}

The study described above characterized the performance of RFID tags under varying orientations on a simulated asset (Styrofoam block) in a closed environment, the GTEM cell. The next step to evaluating the feasibility of RFID technology in a supply chain setting is to investigate the impact of asset position on tag readability. Therefore, assuming that a tag is placed on an asset, we are interested in investigating the impact of the position between the asset and the tag reader on tag readability (i.e. error rates associated with reading tags).

In this study we migrated from the GTEM cell to a test room. This was because the GTEM cell is too detached from real world environments since it provides an environment that is approximately free of extraneous interference. However, in order to eliminate any sources of internal interference all the furniture in the test room was removed. The rationale for removing all furniture from the test room was to establish a baseline for tag readability across a given frequency range using a fixed signal source. Once this baseline has been established it can be applied to a larger set of studies to determine the effect extraneous sources of noise might have on the readability of RFID tags. 


\subsection{Experimental Set-up}

\subsubsection{Apparatus}

The apparatus utilized in this study involved the test environment (i.e. an empty room), an automated measuring system designed as an industrial robot (i.e. the RAMS system), the modified Alien ${ }^{\mathrm{TM}} 9780$ tag reader, two types of RFID tags namely Tag A and Tag C, and a host computer. We proceed by briefly describing the test environment and the RAMS system. Please see section 4.2.1 for descriptions of the other apparatus.

Tests were conducted in a research lab without any furniture. Although the lab was empty for this study it is located in an active research facility with several external sources of potential interference. These include computer equipment from neighboring research labs, a server room, and other random sources of noise that is caused by people walking by and talking on cell phones. The door to the test room was closed during all experimentation, in order to minimize the effects of external human and electrical interference during the experiments. Additionally, there were potential sources of interference within the test room such as electrical conduit, Ethernet, light fixtures, automated temperature management, sprinkler systems, and residual interference from the RAMS system (described below).

Please see Figure 4A for a depiction of the room and axial layout. The X-axis is the length, Y-axis the width, and the $Z$-axis the height. The dimensions of the room and the scanning space utilized are also presented in Figure $4 \mathrm{~B}$. The Alien $^{\mathrm{TM}} 9780$ scanner, host computer, and all control hardware for the RAMS system were housed in a separate room adjacent to the lab with projecting connections transferred through the ceiling.

\section{(A) ROOM AND AXIAL LAYOUT}

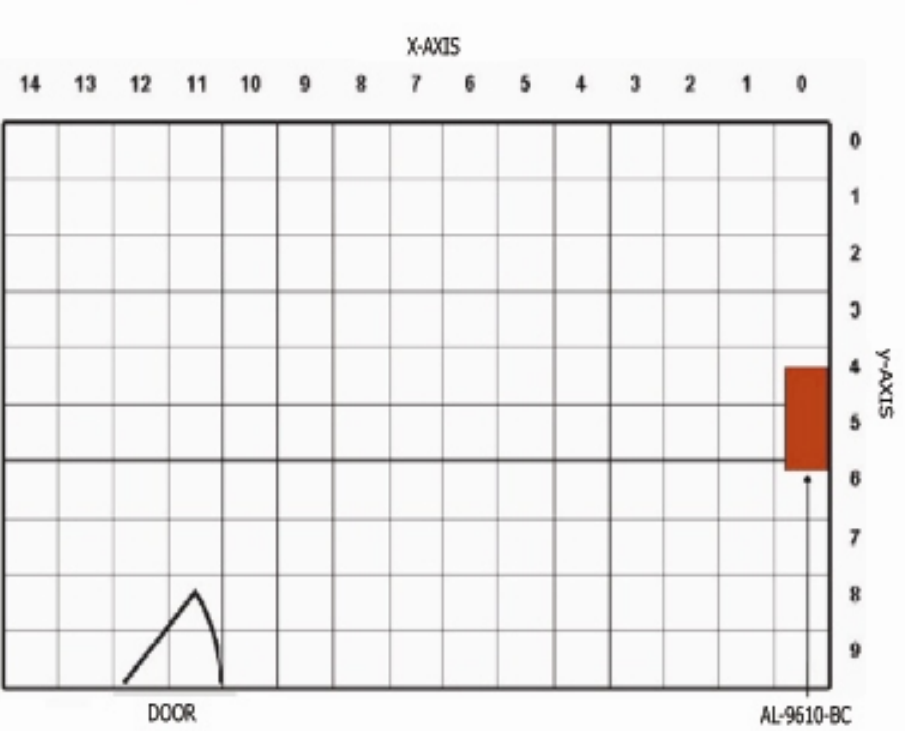

(B) ROOM DIMENSIONS

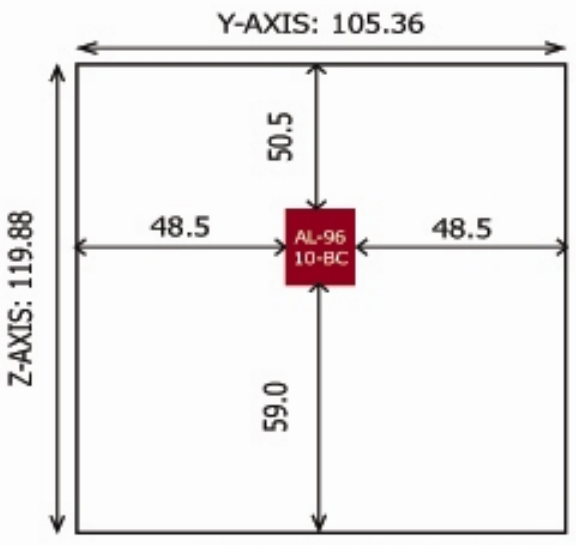

All measurements in inches

Figure 4: Dimensions and layout of test room.

Each grid is $8 \times 8$ sq. Inches

The Rail Automated Measurement System (RAMS) was a robotic measuring system constructed of a low ferrous aluminum frame material. The design of the system involved the use of two thirteen foot long sections of railing material mounted close to the ceiling, parallel with each other on opposite walls that extend the length of the test room. These were the side sections. Two sections of rail material, approximately ten feet long, were attached perpendicular to the side rails constituting the center rail section. Finally, a single section of fiberglass composite railing was used as a vertical arm, and was attached to the center rail section via a double-sided wheel assembly. This design allows for the vertical arm to move smoothly along the center section. To move the different sections (center and vertical only) plastic gears and chains were used in a geared down assembly, to allow for slow and steady movement to prevent excess rocking of the vertical section. Two 6.3V/1.5Amp DC stepper motors, with a $14300 \mathrm{gm}-\mathrm{cm}$ torque, were used to facilitate the moving of the RAMS.

\subsubsection{Procedure}

This study involved the measurement of a single dependent variable, error-rate under variations of three independent variables, the $x, y$, and $z$, co-ordinates of the position of the simulated asset in the room. The characterization of the test room involved conducting scans along the XY-plane for each unit of the Z-axis (7 units spanning 56 inches). Each XY-plane scan comprised of 150 individual points (15 units on the X-axis spanning 120 
inches and 10 units on the Y-axis spanning 80 inches). Overall, 1050 readings were conducted in the test room covering a space of 311.11 cubic feet.

Six RFID tags (3 Tag-A and 3 Tag-C) were tested in this study. Data collection proceeded by first affixing the tag being tested on a Styrofoam block that simulated an asset. The block was then mounted on the measurement arm of the RAMS system. The system was then programmed to automatically move to each area in the grid (see Figure 4A) in a predefined pattern. At each target position in the room the frequency of the signal broadcasted from the scanner was incrementally varied (by $1 \mathrm{MHz}$ ) across a $903 \mathrm{MHz}$ to $927 \mathrm{MHz}$ range. For each frequency at each position 20 read attempts were made. The error rate in reading the tag (ranging from 0 to 1 ) was calculated as the ratio of the number of failures to the number of read attempts. The motors of the RAMS system were shut off before tag reading was initiated.

\subsection{Results}

\subsubsection{Mean Error-Rate for Tag Position}

Figure 5 presents the mean error rates across the $X, Y$, and $Z$ axes. For each sub-plot, in which one of the three independent variables was varied, the mean error rates reported are averaged across the remaining 2 independent variables. We also averaged the error rates for each of the 3 tags of each type.

Figure $5 \mathrm{~A}$ reveals that performance of both tags is poor when placed directly in front of the broadcasting antenna (X $=1$, i.e. < 8"), but improves as the tag moves towards the middle of the room ( $X=2-6$, i.e. 16-48"). Performance steadily drops as the tag moves further away from the antenna (X $=7-10$, i.e. 56-80"). The error rates reach an unacceptable level as the tag hovers around the back wall ( $X=11-14$, i.e. 88-112") where the error rate is in the 0.75-0.85 range. Furthermore, as the tag moves away from the antenna, performance of Tag-A worsens until it is on par with Tag-C.

The results across the $\mathrm{Y}$-axis (Figure $5 \mathrm{~B}$ ) reveal that the error rate is high at the extreme left and right of the antenna $(Y=1-3, Y=7-10$ respectively, i.e. 24" $>Y>56$ "). Performance improves as the position of the tags enters the range of the antenna's field ( $Y=4-6$, i.e. 32" $<Y<48$ "). However, there is a small performance drop (approximately 0.05 units) when the tags are placed directly in front of the antenna ( $Y=5$, i.e. 40"). From the error rates obtained we can conclude that $\operatorname{Tag} A$ significantly outperformed $\operatorname{Tag} \mathrm{C}$. Note that the error rate for Tag-A at $\mathrm{Y}=7$ (further to the right) is on par with that reported for Tag-C when positioned more in line with the antenna $(Y=6)$.

When evaluated across the Z-axis (Figure $5 \mathrm{C}$ ) the results indicate that the error rates for both tag types are higher at the top $(Z=2$, i.e. $<16 ")$ and bottom of the room $(Z=7-8$, i.e. $>56 ")$ with performance at the bottom of the room being worse than that at the top. The error-rates steadily decrease as the tags approach the centre of the room $(Z=3-6$, i.e. 24-48") which is in line with the antenna.

In general tag performance is within the expected trends. In each case, performance of both tags is optimal when the tags are positioned towards the front of the antenna with some minimal (>12") distance between them. Performance is gradually degraded as the tags are positioned towards the extreme ends of each axis - i.e. back of X-axis towards the end of the room, left and right of $Y$ axis, and top and bottom of the Z-axis. We also replicated the findings reported in Experiment 1 where, irrespective of position, Tag-A outperformed Tag-C.
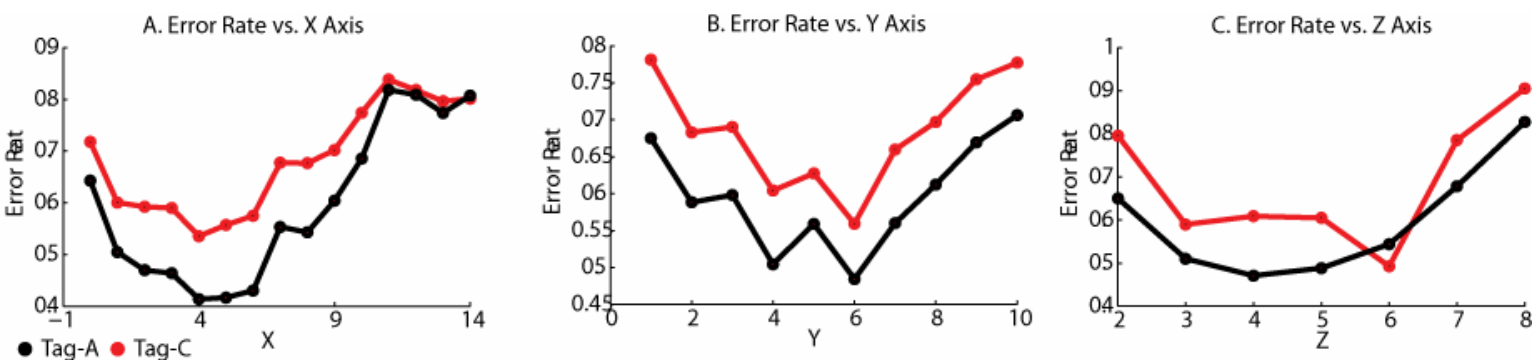

Figure 5: Error rate as a function of position of asset in the test room

\subsubsection{Center Plane Error Rates across Frequency Range}

Since we varied the frequency of the signal from the RFID scanner across the $903 \mathrm{MHz}-927 \mathrm{MHz}$ range for each tag, at each position in the room we have 25 error rate measurements, one for each frequency. However, in a given read cycle, the tag only has to respond to a query on any one of the frequency levels to be successfully read. The results presented in Figure 5 were averaged across the 25 frequency range and are therefore quite conservative. A more useful method to calibrate the performance of the RFID tags would be to consider the difference between the maximum and minimum error rate across the 25 frequencies. Figures $6 \mathrm{~A}$ and $6 \mathrm{~B}$ presents this difference in error rates for Tag-A and Tag-C obtained by scanning the centre $(Z=5)$ plane. Note that while the results presented in 
Figure 6 might seem to be inconsistent with the mean error rates presented in Figure 5, the discrepancy is due to the fact that only the center plane is considered here.
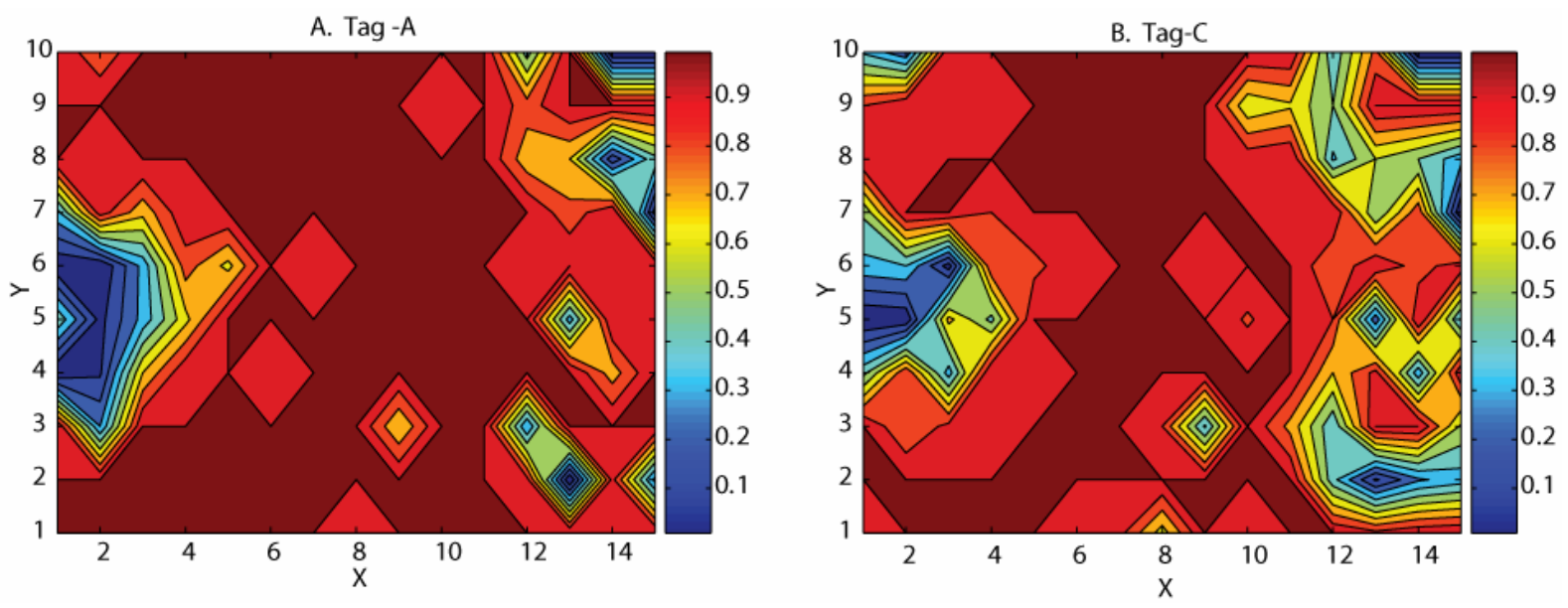

Figure 6: Difference between maximum and minimum error rates across $903-927 \mathrm{MHz}$ frequency bandwidth for the center plane

On the basis of these plots one can divide the test room (along the X-axis) into three zones as specified below.

- Zone I: Front of Antenna. The best performance with respect to readability of the tags can be achieved in this zone. This is because in this area the range of the broadcasted signal is at the maximum.

- Zone II: Mid Room. RFID tags are readable at this area of the room. However, error rates are higher when compared to those obtained in Zone 1. In particular a certain degree of dispersion in reading tags can be noted.

- Zone III: Back Wall. Readability in this zone is less than in Zone 1 but better than Zone 2. This is particularly due to "hot spots" being created due to the presence of the back wall of the room.

\subsection{Discussion Study 2}

As seen by the results presented above even in a simplistic real world environment (without any furniture) the error rates of RFID tags can be quite high. The zonal approach proposed above to manage those errors can be employed in the supply chain setting to optimize interrogating RFID tags. Note also that while the zonal approach proposed here generalizes across tags, there are significant individual differences between tag types. In particular Tag A outperforming Tag $C$ for the latter part of the room (Zone III). Again this needs to be factored in to decision making on implementing RFID in the real world supply chain.

\section{General Discussion}

The increased and somewhat hasty integration of RFID technology into the supply chain has highlighted a host of technical, logistic, and usability issues. While several have been quick to create RFID enabled products, a systematic investigation into the performance and usability issues that occur in the supply chain is lacking. While the research reported in this paper did not explicitly tackle these issues, it signifies a preliminary step into gauging the reliability and overall feasibility of incorporating RFID technology in supply chain environments. By evaluating the reliability in reading RFID tags in two controlled environments a suitable baseline consisting of an upper bound for performance has been established.

With regard to tag characteristics, Study 1 showed that orientation alone can alter the readability of tags. This has implications with regard to how RFID tags are placed on assets that are being tracked. Furthermore, we exposed differences in tag performance with regard to their sensitivity by simulating increasing distances to the reader. Coupling these tag characteristics together, we show that even 30 degrees in orientation and relatively short distances can alter whether a tag can in fact be read by a reader and that furthermore tags differ in their ability to be read based upon these fundamental factors. For total supply chain visibility in a system with multiple members (i.e. multi-vendor, multi-supplier, multi-customer, multi-client, etc.) standardizing reader and tag orientation can mitigate challenges faced with regard to tag characteristics. However implementing such standards in diverse multi-member supply chains is likely to be an intricately complicated coordination and verification challenge. While standardizing on a tag orientation and reader orientation may seem challenging, even more challenging for multi-member supply chains would be standardizing on a specific tag vendor. As shown in this work specifying tag and reader and 
orientation is no guarantee that the distance to the reader is optimal for each tag type or that orientation specified is suitable for all tag types. As demonstrated by our study of 4 tag types, there are significant differences in the performance of the tags with regard to orientation and sensitivity (i.e. ability to be read at varying distances). In a multi-tag supply chain setting, we showed error rates varying quite a bit across these vary factors.

Looking at the environment, our second study took the tag results to a real world setting where reasonable controlled and plausible interference could occur. This study further shows the complications that can arise in implementing RFID beyond tag differences. Within the limited scope of our real world environment and the two tags tested, error rates were quite high under certain conditions and as would be expected differ between the two tags types tested. While a generalization can be made by zoning the interrogation space of the reader (see above), this still does not adequately describe the differences seen between the tags.

\section{Conclusion and Future Work}

We have shown empirical testing of tags and environments in supply chains; we feel it is worth noting that other obstacles in implementation that we did not test can occur. Some of the issues that might reduce the generalizability of the results are that only a handful of different RFID tags were used and did not include current "Gen 2" tags. Another issue of potential concern lies in the fact that only one type of RFID scanner was used and its antenna was only placed at a single location in the test room (for Study 2 ).

A number of extensions can be made to the study to increase its external validity. These include evaluating different types of tags such as tags from the Avery-Dennison (AD) series. Additional experiments that vary the position of the antenna in the room could also be useful. Another useful set of experiments would involve using different types of RFID scanners.

In addition to these variations, the next step towards this research would be to systematically replicate the data collection procedure but with additional assets in the room. By comparing the tag error rates reported here with those collected with a variety of assets in the room, we can make assessments as to the effect the assets have on the tag error rates. Another avenue of research that is being pursued is the impact of different packaging materials on tag readability. By affixing tags on a variety of materials including cardboard, wood, glass, Plexiglas, and metal, we intend to calibrate the effects of the surface material of an asset on tag readability.

It is our view that the fundamental technical obstacles that are currently challenging total asset visibility will likely prevent widespread adoption in the near term. Two major conclusions can be drawn from our studies involving the systematic testing of commercially available RFID tags. First, the generally poor performance of RFID technology in highly controlled environments (a GTEM cell in Study 1 and an empty room in Study 2), questions the applicability of this technology in real world supply chains that are undoubtedly susceptible to several additional sources of interference. Second, the fact that orientation of a tag on an asset has such an enormous impact on readability, raises several logistic issues related to the placement of tags on an asset and the orientation of the asset while it travels down the supply chain. Could it be the case that the best solution is to place multiple tags, with the same information, on the asset? Should multiple antennas that ostensibly cover every square inch of space be deployed all along the supply chain? Are these options financially feasible and do they mark significant technological improvements that extends above and beyond the placement of barcode readers at every step of the supply chain?

While answers to these questions are beyond the scope of this paper, it is our hope that the results we have discussed here coupled with findings from our future studies will inform RFID adoption in supply chains. It is with time, scientific rigor, and engineering innovation that that many of the current RFID challenges will be overcome.

\section{Acknowledgments}

The authors would like to acknowledge past and present members of the RFID Consortium at the FedEx Institute of Technology at the University of Memphis. In particular we would like to thank Divya Choudhary, Srikant Chari, Jason Keen, Suresh Susarla, and Eddy Ochieng for their valuable contributions to this research. We also gratefully acknowledge Tsuyoshi Ohashi, Takuya Nagai, and Rodney Loyd from Brother Industries, Ltd, Joe Branc and Joel Stanfield from Steelcase, Inc., and Miley Ainsworth of FedEx for their sustained guidance and support. We would like to thank IntelliMark for donating equipment and volunteering consulting services during the initial phase of this project.

This research was conducted with funds and consulting assistance provided by Brother Industries Ltd and Steelcase Inc. Any opinions, findings and conclusions or recommendations expressed in this paper are those of the authors and do not necessarily reflect the views of Brother Industries Ltd or Steelcase Inc.

\section{References}

[1] Alien Technologies. (2006). [Online]. Available: http://www.alientechnology.com/ 
[2] ALR 9780 data sheet. (2006). [Online]. Available: http://www.alientechnology.com.

[3] R. Angeles, RFID Technologies: Supply-Chain Applications and Implementation Issues, Information Systems Management, vol. 22, pp. 51-65, 2005.

[4] Z. Asif and M. Mandviwalla, Integrating the Supply Chain with RFID: a technical and business analysis, Communications of the Association for Information Systems, vol. 15, pp. 393-427, 2005.

[5] M. Attaran, RFID: an enabler of supply chain operations, Supply Chain Management: An International Journal, vol. 12, pp. 249-257, 2007.

[6] M. Brandel. (2003, December) Smart tags, high costs, ComputerWorld [Online] Available: http://www.compu terworld.com/softwaretopics/erp/story/0,10801,88130,00.html.

[7] B. Brewin, (2003, October), RFID users differ on standards, ComputerWorld [Online]. Available: http://ww w.computerworld.com/softwaretopics/erp/story/0,10801,86486,00.html.

[8] H. Chae, T. Lee, and H. Peter, Situation Aware RFID system: Evaluating abnormal behavior detecting approach, in Proc. SEUSWCCIA' 06, April 2006, pp. 115-120.

[9] G. Chang, A Feasible Security Mechanism for Low Cost RFID Tags, in Proc. Intl. Conf. on Mobile Business (ICMB'05), July 2005, pp. 675- 677.

[10] D.W. Engels and S.E. Sarma, The Reader Collision Problem, white paper MITAUTOID- WH-007, Auto-ID Center, Nov. 2001.

[11] C. Floerkemeier and M. Lampe, Issues with RFID usage in ubiquitous computing applications, in Proc. 2nd Intl. Conf. Pervasive Computing: LNCS No. 3001, Austria, 2004, pp. 188-193.

[12] C. Floerkemeier and M. Wille, Comparison of Transmission Schemes for Framed ALOHA based RFID Protocols, in Proc. Intl. Symposium on Applications and the Internet Workshops (SAINT 2006), pp. 92-97, January 2006.

[13] S. Garfinkel, An RFID Bill of Rights, Technology Rev., Oct. 2002, p. 35.

[14] G. Goth, RFID: Not Quite Prime Time, But Dawdle at Your Own Risk, IEEE Distributed Systems Online, pp. 3, February 2005.

[15] S. L. Garfinkel, A. Juels, and R. Pappu, RFID Privacy: An Overview of Problems and Proposed Solutions, IEEE Security and Privacy, pp. 34- 43, May 2005.

[16] J.-L. Hou and H. Chih-Hao, Quantitative performance evaluation of RFID applications in the supply chain of the printing industry, Industrial Management \& Data Systems, vol. 106, pp. 96-120, 2006.

[17] T-W. Hwang, B-G. Lee, Y. S. Kim, D. Y. Suh, and J. S. Kim, Improved Anti-collision Scheme for High Speed Identification in RFID System, in Proc. 1st Intl. Conf. on Innovative Computing, Information and Control - Volume II (ICICIC'06), pp. 449-452, August 2006.

[18] J. Korhonen, T. Ojala, M. Klemola, and P. Vaananen, mTag - Architecture for Discovering Location Specific Mobile Web Services Using RFID and Its Evaluation with Two Case Studies, in Proc. AICTICIW' 06, pp. 191, February 2006.

[19] A. Lefebvre, E. Lefebvre, Y. Bendavid, S. F. Wamba, RFID as an Enabler of B-to-B e-Commerce and Its Impact on Business Processes: A Pilot Study of a Supply Chain in the Retail Industry, in Proc. 39th Annual Hawaii Intl. Conf. on System Sciences (HICSS'06), pp. 104a, January 2006.

[20] K. S. Leong, M. L. Ng, and P. H. Cole, Positioning Analysis of Multiple Antennas in a Dense RFID Reader Environment, in Proc.Intl. Symposium on Applications and the Internet Workshops (SAINT 2006), pp. 56-59, January 2006.

[21] K. Michael and L. McCathie, The pros and cons of RFID in supply chain management, presented at International Conference on Mobile Business (ICMB '05), 2005.

[22] J. M. Myerson, RFID in the Supply Chain: A Guide to Selection and Implementation: AUERBACH, 2006.

[23] B. Nath, F. Reynolds, and R. Want, RFID Technology and Applications, IEEE Pervasive Computing, January 2006, pp. 22-24.

[24] M. O'Halloran and M. Glavin, RFID Patient Tagging and Database System, in Proc. ICNICONSMCL'06, April 2006, pp. 162.

[25] T. Phillips, T. Karygiannis, and R. Huhn, Security Standards for the RFID Market, IEEE Security and Privacy, pp. 85-89, November 2005.

[26] K. V. S. Rao, P. V. Nikitin, and S. F. Lam, Impedance Matching Concepts in RFID Transponder Design, in Proc. Fourth IEEE Workshop on Automatic Identification Advanced Technologies (AutolD'05), pp. 39-42, October 2005.

[27] M. R. Rieback, B. Crispo, and A. S. Tanenbaum , The Evolution of RFID Security, IEEE Pervasive Computing, pp. 62-69, January 2006

[28] J. Rothfeder, What's Wrong with RFID?, in CIO Insight, 2004.

[29] G. Roussos, Enabling RFID in Retail, Computer, pp. 25-30, March 2006.

[30] C. Sellitto, S. Burgess, and P. Hawking, Information quality attributes associated with RFID-derived benefits in the retail supply chain, International Journal of Retail \& Distribution Management, vol. 35, pp. 69-87, 2007.

[31] S. Singh, Low Cost Object Identification in RFID via Dynamic Markov Chain \& Two Time Scale SPSA, in Proc.Intl. Symposium on Applications and the Internet Workshops (SAINT 2006), pp. 64-67, January 2006.

[32] S. Wang, W. Chen, C. Ong, L. Liu, and Y. Chuang, RFID Application in Hospitals: A Case Study on a Demonstration RFID Project in a Taiwan Hospital, in Proc. 39th Annual Hawaii Intl. Conf. on System Sciences (HICSS'06), pp. 184a, January 2006.

[33] R. Want. An Introduction to RFID Technology, IEEE Pervasive Computing, January 2006, pp. 25-33.

[34] I. H. Yasuura and D. Hagiwara, Systematic Error Detection for RFID Reliability, in Proc. 1st Intl. Conf. on Availability, Reliability and Security (ARES'06) , pp. 280-286, April 2006. 
[35] L. Zhang and Z. Wang, Integration of RFID into Wireless Sensor Networks: Architectures, Opportunities and Challenging Problems, in Proc. 5th Intl. Conf. on Grid and Cooperative Computing Workshops, 2006, pp. 463469. 